\title{
Ownership changes and access to external financing
}

\author{
Anzhela Knyazeva ${ }^{a, *}$, Diana Knyazeva ${ }^{a}$, Joseph Stiglitz ${ }^{b}$ \\ ${ }^{a}$ William E. Simon Graduate School of Business Administration, University of Rochester, Rochester, NY 14627, USA \\ ${ }^{\mathrm{b}}$ Columbia Business School, Columbia University, New York, NY 10027, USA
}

\section{A R T I C L E I N F O}

\section{Article history:}

Received 20 July 2008

Accepted 29 December 2008

Available online 18 January 2009

\section{JEL classification:}

G38

G31

L33

Keywords:

Privatization

Ownership

Access to credit

Performance

Investment

\begin{abstract}
A B S T R A C T
This paper examines access to external financing in the privatization context and provides new evidence on the effects of financing constraints on performance and investment. Ownership reforms increase firms' reliance on external financing. Empirically, performance and investment changes around ownership reforms are increasing in country-level measures of access to credit. The presence of a severe prior public financing constraint contributes to stronger investment growth after privatization. Privatized enterprises do not outperform publicly owned industries, all else given. Our analyses rely on new international sector- and firm-level data and correct for potential endogeneity of ownership changes.
\end{abstract}

(c) 2009 Elsevier B.V. All rights reserved.

\section{Introduction}

The relation between ownership structure and performance has long been an important issue in theoretical and empirical corporate finance. Private ownership and ownership reforms have been shown to improve firm performance through aligning the incentives of managers and shareholders and increasing the efficiency of investment decisions. A change in the sources of funding and the extent of reliance on external financing is an important implication of ownership reforms mostly overlooked in earlier analysis. To our knowledge this study is the first systematic attempt to examine the role of access to external financing for performance and investment changes following privatizations. We find a significant effect of country-level measures of access to credit on performance and growth around ownership reforms over different time horizons. We also find that prior constraints are associated with significantly higher investment growth following ownership changes. However, comparisons of privatized industries with similar publicly owned industries reveal that a shift to private ownership alone does not lead to better performance. Our tests employ new international telecommunications sector data as well as

\footnotetext{
* Corresponding author. Tel.: +1 585275 3102; fax: +1 5852731140.

E-mail addresses: anzhela.knyazeva@simon.rochester.edu (A. Knyazeva), diana. knyazeva@simon.rochester.edu (D. Knyazeva), jes322@columbia.edu (J. Stiglitz).
}

firm-level data for multiple sectors, and they correct for selection bias and endogeneity.

This paper provides novel evidence on the interaction between operating decisions and financing. Access to external financing is hypothesized to have a large effect on capital-intensive companies that become more dependent on private funding of new investment projects after a change from public to private ownership. This paper also sheds more light on the issue of investment and soft budget constraints. According to a prevalent view, inefficient investment in publicly owned enterprises can be curbed by switching to a hard budget constraint under private ownership. Our findings instead underline the significance of past financing constraints and increased access to external financing for improvements in performance and investment growth following a change from public to private ownership.

Focusing on a sample of capital-intensive industries, we analyze the effects of access to financing before and after ownership changes to distinguish between the financing dimension and other factors that contribute to the impact of ownership reforms on performance. We examine the effects of access to external financing and the relaxation of prior public sector financing constraints on performance around ownership changes. We argue that access to external financing improves the ability of new owners to finance investment projects and strengthens performance. Empirically, we find a significant positive effect of access to credit on changes in performance and investment around ownership reforms. 
Further, publicly owned enterprises with more severe constraints on access to financing due to low debt capacity of the public sector experience faster investment growth after the ownership change.

We also empirically evaluate the relative importance of access to financing and ownership changes for long-term performance. Similar to Knyazeva et al. (2006), we account for selection and endogeneity issues involving the ownership change decision. While access to external financing has a positive effect on long-term performance changes, privatized industries do not perform better than industries remaining public during the same period.

Our paper is related to existing work on institutions and financial development. Rajan and Zingales (1998) find that growth in industries dependent on external capital is higher in countries with better capital markets. Demirguc-Kunt and Maksimovic (1998) conclude that firms are able to finance more growth through external financing in countries with better financial development and legal environment quality. Beck and Levine (2002) demonstrate the relevance of overall financial development for growth and the formation of new establishments. Beck et al. (2006) find a positive effect of financial intermediary development on firm size. Unlike the cited works, this paper considers the effects of prior financing constraints and current access to credit on performance around ownership changes. We ask which of the two factors is more important for performance and how access to external financing interacts with major ownership changes.

Our study also relates to the privatization literature. Existing papers perform pre- and post-privatization comparisons and find positive effects of privatization, competition, and regulation on performance (Dewenter and Malatesta, 2001; Megginson et al., 1994; D'Souza and Megginson, 1999; D'Souza etal., 2001; Boubakri and Cosset, 1998; Wallsten, 2001; Bortolotti et al., 2002). For instance, Boubakri et al. (2005) show post-privatization improvements in efficiency and profitability and a link among property rights, stock market turnover, and efficiency gains. Similarly, state-owned banks exhibit lower profitability, efficiency, and asset quality, and they engage in less prudent lending practices (Lin and Zhang, 2009; Jia, 2009; Iannotta et al., 2007).

Unlike other work, this paper focuses on the effects of access to credit and past financing constraints on post-privatization performance. We contribute to existing literature by showing a link between access to external financing, particularly through the credit channel, and performance and investment around ownership changes. Our analyses disentangle the ownership change decision, performance consequences of ownership changes, and differences in long-term performance of publicly owned and privatized industries to draw conclusions about the role of access to financing before and after ownership reforms for performance and operating decisions. We also find that privatized industries do not outperform public sectors after controlling for endogeneity.

This paper uses a comprehensive telecommunications sector data set. The chosen setting offers several advantages: increased relevance of external financing due to the industry's capital intensity, fewer concerns about the endogeneity of country-level financing conditions to a single sector's performance, and greater homogeneity and comparability of data across countries. Further, telecommunications sector findings are supplemented with firmlevel evidence obtained in a multi-sector sample. Our empirical methodology accounts for the endogeneity of ownership reforms.

Ownership reforms trigger major shifts in the sources of funding for new projects. Our first hypothesis concerns the role of access to credit for post-privatization performance changes. Recently privatized firms lack a reputation in capital markets and face potentially significant information asymmetries. Due to lower information sensitivity of debt, the lending channel would appear to be an important method of accessing additional external financing for such firms. We argue that the availability of credit has a favorable effect on performance around ownership changes. Developed credit markets promote more efficient allocation of capital to new investment projects. Recently privatized firms that lack internal cash flow to finance value-increasing projects can avoid underinvestment due to increased availability and (or) lower cost of credit. Therefore, our first hypothesis is that firms that switched to private ownership exhibit stronger performance improvements and higher investment growth in environments with better access to bank credit.

An alternative prediction arises from the argument that financial market monitoring limits managerial slack and empire building as well as helps eliminate inefficient investments undertaken prior to privatization, resulting in better performance of borrower firms. Hence, while performance would improve at a faster rate in the presence of developed credit markets, investment would decrease or grow more slowly. We consider the effects of credit on performance and investment changes to distinguish empirically between our main hypothesis and this competing prediction.

Our second hypothesis relates financing constraints prior to privatization to performance changes after ownership reforms. Reduced borrowing capacity of the government can limit the funding available to state-owned entities, potentially resulting in underinvestment in value-improving projects while the industries are predominantly publicly owned. State-owned enterprises that experience internal cash flow shortfalls have less financial flexibility if the public sector is subjected to financing constraints. Enterprises that were subjected to public sector financing constraints prior to privatization might realize larger gains from a shift to private ownership. They are expected to benefit more from the financing obtained during the privatization transaction and from raising external financing as a privately owned company, resulting in higher growth after the ownership reform. Previously unconstrained public enterprises are expected to gain less from the ownership change. Therefore, our second hypothesis is that performance and investment changes around ownership reforms are increasing in prior financing constraint.

We also ask whether privatized industries outperform comparable publicly owned industries. Several possible considerations arise. According to the soft budget constraint argument, the switch to private ownership increases investment efficiency and benefits performance by eliminating value-destroying investments. According to an alternative view, if the costs of the reforms are factored in, privatized industries need not outperform publicly owned industries, at least in the short to medium run (Knyazeva et al., 2006).

However, the relation of ownership change and performance can be confounded by the opposite effects observed for different subsamples of privatized industries. Instead of examining the overall effects of ownership reforms on performance, this paper attempts to identify industries that benefit from a change to private ownership and those that fail to realize improvements. For previously constrained industries, the switch to private ownership is expected to increase access to financing and, by consequence, performance. Privatization need not benefit previously unconstrained enterprises. Reputation building in capital markets takes time, while unconstrained governmentowned enterprises receive continuous funding. In addition, undervalued deals are more vulnerable to the risk of reversal of privatization, which increases the cost of capital (Hoff and Stiglitz, 2004). We therefore examine subsamples based on prior constraint, current access to external financing, and institutional environment to disentangle the positive and negative effects of privatization. 
The remainder of the paper is organized as follows. Section 2 describes the data and methodology. Section 3 presents the results. Section 4 concludes.

\section{Data}

\subsection{Sample}

The first set of tests examines performance and investment dynamics around ownership changes. We use a cross-country sample of telecommunications industry privatizations. Privatization reform data are obtained from Guillen et al. (2004) for 19871999. Certain control variable definitions require data availability for 1982-1984. Industry performance and investment variables are obtained from International Telecommunication Union World Telecommunications Indicators. The second set of tests analyzes long-term performance changes in the full sample of privatized and publicly owned industries.

We focus on the telecommunications sector for several reasons. First, capital intensity of the telecommunications sector heightens the role of access to external financing and prior financing constraints for investment and performance. The findings can inform inference about other capital-intensive industries. Second, country-level regressions of economic growth on financial development examined in earlier work are susceptible to causality concerns. Our use of performance and investment data for a single sector mitigates the issue of endogeneity of country-level access to capital measures. Third, data on operating decisions and performance within a single sector are more comparable across countries.

The final set of tests corroborates the main results in a multisector sample of European privatization deals conducted in 1989-2006. Data on years of privatization and privatized stakes are obtained from Privatization Barometer. Firm-level privatization data are matched to Amadeus data on financial characteristics of privatized companies.

\subsection{Variables}

Telecommunications sector analyses use several dependent variables. All variables are expressed as changes. The first two measures characterize growth in output and availability of service. Total subscriber density is defined as the number of total telephone subscribers per hundred inhabitants. The measure accounts for possible substitution of mobile service for fixed line service. However, it could falsely attribute the spike in phone service due to cell phone usage to the effect of ownership reforms. Therefore, we also use fixed phone line density, defined as the number of fixed lines per hundred inhabitants. The third measure is total capacity (telephone line connective capacity scaled by population). The fourth measure is sector investment, defined as the ratio of telecommunications investment to gross domestic product (GDP). Due to data constraints, we resort to aggregate sector-level data on annual investment expenditure. Given the high concentration of the telephone service industry, particularly in the immediate aftermath of ownership reforms, changes in sector investment should be a reasonably accurate reflection of the trends in investment of recently privatized telecom providers. The fifth measure characterizes sector revenues (the ratio of annual telecommunications revenue to GDP).

Firm-level analyses use profitability variables, including the ratio of earnings before interest and taxes (EBIT) to total assets and profit margin, to measure operating performance more directly.

One of the main explanatory variables in this study is access to credit; particularly, bank credit. Bank credit plays a significant role in a number of developed countries in the sample, but especially so in countries with less developed equity markets. Recently privatized enterprises are likely to face higher information asymmetries than companies with an established reputation in the capital markets. Bank credit is expected to be an important method of raising external financing for recently privatized companies. The bank credit measure can also be used to distinguish the main hypothesis from the alternative soft budget constraint prediction. As delegated monitors, banks can oversee the management to prevent overinvestment. If recently privatized companies have valuedestroying investments as a result of past soft budget constraints, bank credit should have a positive effect on performance but a negative effect on investment growth. However, if the access to external financing hypothesis holds, bank credit should have a positive effect on both performance and investment. The variable is defined as the share of domestic bank credit in GDP. For robustness, we consider the share of domestic private sector credit in GDP. We also examine the sensitivity of access to credit results to the inclusion of equity market development measures. ${ }^{1}$

Due to the scarcity of firm-level data on pre-privatization financing constraints, we define prior constraint based on the past debt burden of the government. High debt levels limit the government's borrowing capacity and increase interest payments, hindering the state's ability to finance projects in capital-intensive state-owned industries. Similar to country-level measures of access to credit, our prior constraint variable has the advantage of being relatively exogenous to future performance of an individual sector. The variable is defined as the average share of public and publicly guaranteed debt in GDP prior to the ownership change. The share of external debt in GDP is used in robustness checks. In addition, we use the presence of a sovereign debt rating prior to privatization to proxy for past availability and cost of government debt financing.

Several controls are included. Competition and the presence of an independent regulator are expected to improve performance ( Wallsten, 2001; Li and $\mathrm{Xu}, 2004$ ). The competition index provided by Li and Xu (2004) and Estache and Goicoechea (2005) is a categorical variable that varies among monopoly, duopoly, partial competition, and extensive competition. We also control for past performance, country income, and legal origin (based on La Porta et al., 1998). The year of privatization is included to account for learning about privatization design and possible technological shifts. For robustness we control for the scope of the ownership change (full or partial privatization) to capture variation in incentives. Firm-level performance analyses include controls for firm size, the share of tangible assets in total assets, average industry performance, the number of years since privatization, percentage privatized, and year dummies. Because Frydman et al. (1999) find that privatization gains are realized with outsider but not insider owners, we also control for post-privatization ownership structure in firm-level tests (Amadeus). Other robustness checks introduce controls for privatization method and value of privatization transactions (World Bank Privatization Database and Privatization Barometer). Table 1 reports descriptive statistics for the main variables.

\subsection{Methodology}

We analyze performance changes over several time horizons. First, we examine changes in performance in the three years after the ownership reform (years +1 to +3 ) relative to three years before the ownership reform (years -3 to -1 ). The ownership reform year (year 0 ) is excluded. Second, we analyze the effects of ownership

\footnotetext{
${ }^{1}$ Access to bank credit in a given country is arguably exogenous to the performance of a single sector or firm, whereas stock market liquidity is more likely to be affected by countrywide share issue privatizations (Bortolotti et al., 2007).
} 
changes and access to financing on long-term changes in performance over fixed windows (1987-1989 to 2000-2002). Third, we estimate panel data regressions of performance changes using all available observations (without limiting the period of analysis to three years after the reform). Panel data regressions include the number of years since privatization to control for the time-varying effect of past ownership changes.

If performance is persistent, some industries could systematically outperform others. To address this, we either examine changes in performance or include fixed effects and control for average industry performance. The downside of fixed effects esti- mation is the exclusion of time-invariant characteristics such as pre-privatization financing constraint, legal environment quality, and privatization method.

Sectors or firms with certain unobserved time-invariant characteristics could be targeted for privatization (Gupta, 2005). Selfselection into the ownership reform sample would preclude valid inference. To account for this, we include a set of country-, sector-, or firm-level controls that could be correlated with post-privatization performance, including initial performance. A selection model is used to analyze performance changes after privatization. We use treatment effects estimation of the effect of privatization

Table 1

Descriptive statistics of the main variables.

\begin{tabular}{|c|c|c|c|c|}
\hline Variable & $N$ & Mean & Median & S.D. \\
\hline \multicolumn{5}{|l|}{ Sector-level analysis } \\
\hline \multicolumn{5}{|l|}{ Privatized industries } \\
\hline$\Delta$ Output(I) & 54 & 2.233 & 1.424 & 2.736 \\
\hline$\Delta$ Output(II) & 53 & 4.440 & 1.951 & 6.094 \\
\hline$\Delta$ Capacity & 50 & 3.159 & 2.186 & 3.510 \\
\hline$\Delta$ Invest & 39 & 0.651 & 0.499 & 0.733 \\
\hline$\Delta$ Revenue & 38 & 0.834 & 0.816 & 0.727 \\
\hline Access to credit & 54 & 0.373 & 0.259 & 0.343 \\
\hline Prior constraint & 54 & 0.679 & 0.462 & 0.831 \\
\hline Independent regulator & 54 & 0.093 & 0.000 & 0.293 \\
\hline Competition & 54 & 0.861 & 0.065 & 1.176 \\
\hline Geographic area & 54 & 11.834 & 12.234 & 2.289 \\
\hline Private credit & 54 & 0.262 & 0.168 & 0.270 \\
\hline Equity market cap & 31 & 0.263 & 0.126 & 0.437 \\
\hline Prior external debt constraint & 54 & 0.822 & 0.592 & 0.932 \\
\hline Semi-autonomous regulator & 54 & 0.130 & 0.000 & 0.339 \\
\hline Scope of privatization & 44 & 7.702 & 5.731 & 6.971 \\
\hline \multicolumn{5}{|l|}{ All industries } \\
\hline Privatization & 141 & 0.383 & 0.000 & 0.488 \\
\hline Full privatization & 141 & 0.071 & 0.000 & 0.258 \\
\hline Initial output & 141 & 5.228 & 2.136 & 7.924 \\
\hline Initial country income & 141 & 6.896 & 6.890 & 1.133 \\
\hline French legal origin & 141 & 0.482 & 0.000 & 0.501 \\
\hline Initial population & 141 & 15.226 & 15.353 & 1.942 \\
\hline Geographic latitude & 141 & 0.255 & 0.200 & 0.179 \\
\hline Catholic religion & 141 & 30.682 & 16.900 & 35.165 \\
\hline Initial budget balance & 86 & -4.776 & -4.030 & 6.701 \\
\hline \multicolumn{5}{|l|}{ Firm-level analysis } \\
\hline$\Delta \mathrm{EBIT} / \mathrm{A}$ & 945 & -0.055 & 0.245 & 10.834 \\
\hline$\Delta$ Profit margin & 792 & 3.093 & 1.390 & 20.843 \\
\hline$\Delta$ Income per capita & 945 & 0.159 & 0.130 & 0.117 \\
\hline GDP growth & 945 & 3.368 & 3.344 & 2.286 \\
\hline Years since privatization & 945 & 4.415 & 4.000 & 2.971 \\
\hline Size & 945 & 0.120 & 0.194 & 2.700 \\
\hline Tangible assets & 945 & 0.465 & 0.469 & 0.280 \\
\hline Debt/A & 859 & 0.524 & 0.531 & 0.277 \\
\hline Percent privatized & 945 & 64.394 & 70.000 & 28.429 \\
\hline Transaction value & 729 & 0.131 & 0.013 & 0.446 \\
\hline
\end{tabular}

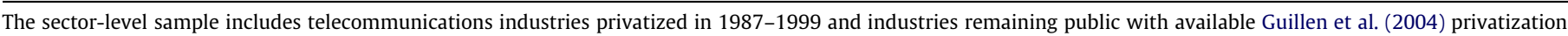

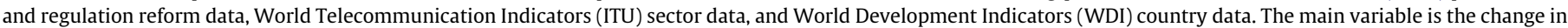

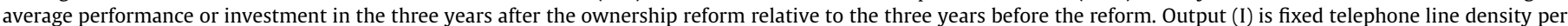

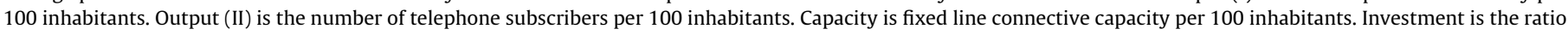

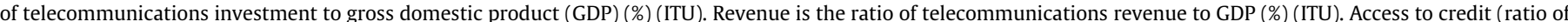

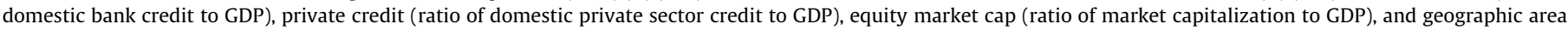

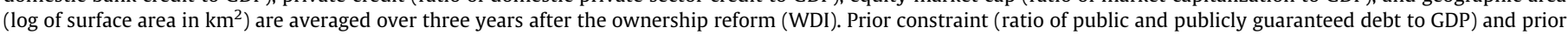

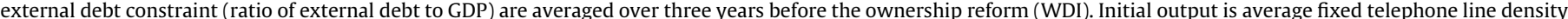

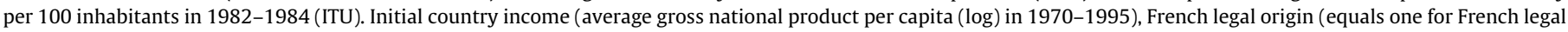

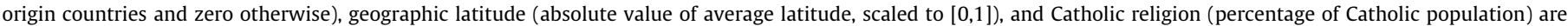

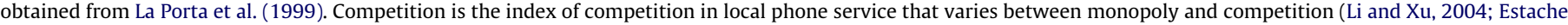

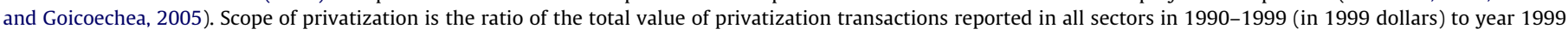

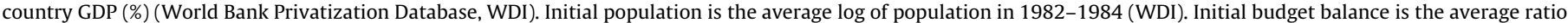
of budget balance to GDP (\%) in 1982-1984 (WDI).

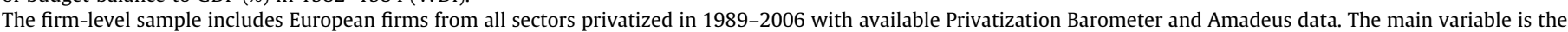

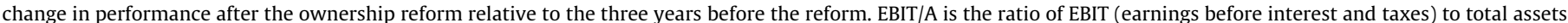

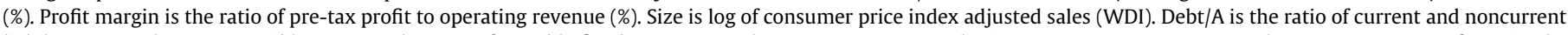

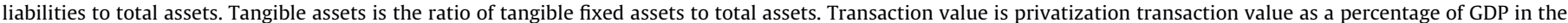

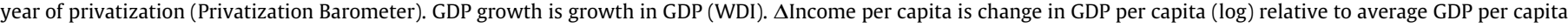
$(\log )$ in the three years before the reform (WDI). 
on performance changes to address the possible endogeneity of privatization. The resulting estimates are consistent. Treatment effects estimation is more efficient than instrumental variables esti- mation. Further, as fixed effects filter out unobserved group-level heterogeneity, fixed effects estimation partly addresses the endogeneity problem.

Table 2

Privatization decision and choice of privatization type.

\begin{tabular}{|c|c|c|c|c|c|}
\hline & $\begin{array}{l}\operatorname{Pr} \text { (Privatization) } \\
\text { I }\end{array}$ & $\begin{array}{l}\text { Pr (Privatization) } \\
\text { II }\end{array}$ & $\begin{array}{l}\operatorname{Pr} \text { (Full privatization) } \\
\text { III }\end{array}$ & $\begin{array}{l}\text { Privatization type } \\
\text { (full, majority, minority, none) } \\
\text { IV }\end{array}$ & $\begin{array}{l}\text { Privatization type } \\
\text { (full versus partial) } \\
\text { V }\end{array}$ \\
\hline Initial output & $\begin{array}{l}-0.048^{*} \\
(0.02)\end{array}$ & $\begin{array}{l}-0.080^{*} \\
(0.04)\end{array}$ & $\begin{array}{l}-0.999^{* * *} \\
(0.31)_{* * *}\end{array}$ & $\begin{array}{l}-0.122^{* *} \\
(0.05)^{* *}\end{array}$ & $\begin{array}{l}-1.271^{* * *} \\
(0.38)^{* * *}\end{array}$ \\
\hline Initial country income & $\begin{array}{l}0.033 \\
(0.15)\end{array}$ & $\begin{array}{l}0.343 \\
(0.22)\end{array}$ & $\begin{array}{l}3.668 \\
(0.84)_{* * *}\end{array}$ & $\begin{array}{l}0.480 \\
(0.21)\end{array}$ & $\begin{array}{l}4.628 \\
(1.06)\end{array}$ \\
\hline Geographic latitude & $\begin{array}{l}-0.015 \\
(0.85)_{* * *}\end{array}$ & $\begin{array}{l}-1.083 \\
(1.28)_{* * *}\end{array}$ & $\begin{array}{l}8.592^{* * *} \\
(2.35)_{* * *}\end{array}$ & $\begin{array}{l}0.071 \\
(1.16)\end{array}$ & $\begin{array}{l}13.692^{* * *} \\
(3.26)^{* * *}\end{array}$ \\
\hline Catholic religion & $\begin{array}{l}0.015^{* * *} \\
(0.0)\end{array}$ & $\begin{array}{l}0.016 \\
(0.01)\end{array}$ & $\begin{array}{l}0.093^{* * *} \\
(0.02)\end{array}$ & $\begin{array}{l}0.023^{* * *} \\
(0.01)\end{array}$ & $\begin{array}{l}0.113^{* * *} \\
(0.03)\end{array}$ \\
\hline French legal origin & $\begin{array}{l}-0.817^{* * *} \\
(0.26)\end{array}$ & $\begin{array}{l}-0.834^{* *} \\
(0.35)_{* *}\end{array}$ & $\begin{array}{l}-4.921^{* * *} \\
(1.32)_{* * *}\end{array}$ & $\begin{array}{l}-0.732^{* *} \\
(0.34)_{* *}\end{array}$ & $\begin{array}{l}-5.652^{* * *} \\
(1.70)_{* * *}\end{array}$ \\
\hline Initial population & $\begin{array}{l}0.075 \\
(0.07)\end{array}$ & $\begin{array}{l}0.170^{* *} \\
(0.09)\end{array}$ & $\begin{array}{l}0.989 \\
(0.26)\end{array}$ & $\begin{array}{l}0.185^{* *} \\
(0.07)\end{array}$ & $\begin{array}{l}1.179^{* * *} \\
(0.34)\end{array}$ \\
\hline $\begin{array}{l}\text { Initial budget } \\
\text { balance }\end{array}$ & & $\begin{array}{l}-0.038 \\
(0.03)\end{array}$ & $\begin{array}{l}-0.175^{* * *} \\
(0.04)\end{array}$ & $\begin{array}{l}-0.037^{* *} \\
(0.02)\end{array}$ & $\begin{array}{l}-0.218^{* * *} \\
(0.06)\end{array}$ \\
\hline Number of observations & 141 & 86 & 86 & 86 & 86 \\
\hline Pseudo $R^{2}$ & 0.12 & 0.19 & 0.70 & 0.18 & \\
\hline
\end{tabular}

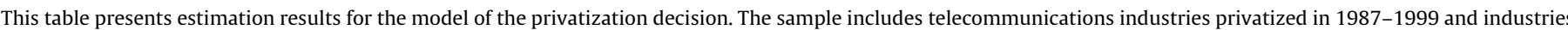

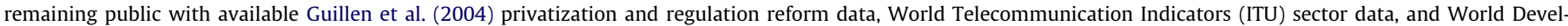

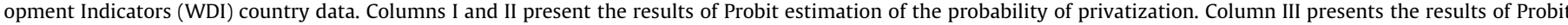

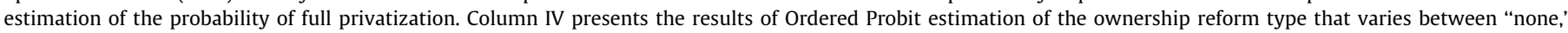

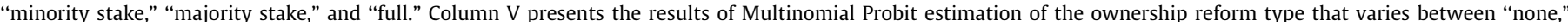

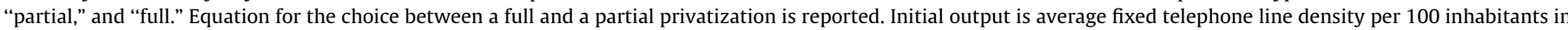

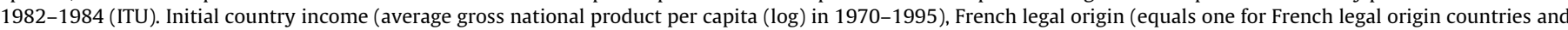

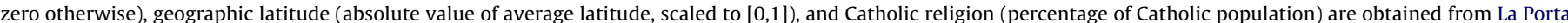

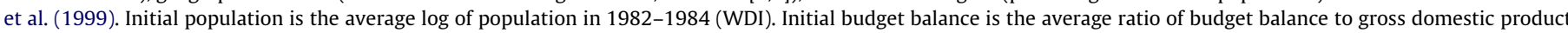

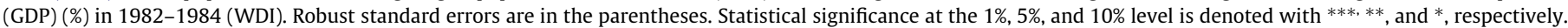

Table 3

Performance around ownership changes (sectoral study).

\begin{tabular}{|c|c|c|c|c|c|c|}
\hline & $\begin{array}{l}\Delta \text { Output(I) } \\
\text { I }\end{array}$ & $\begin{array}{l}\Delta \text { Output(I) } \\
\text { II }\end{array}$ & $\begin{array}{l}\Delta \text { Output(II) } \\
\text { III }\end{array}$ & $\begin{array}{l}\Delta \text { Capacity } \\
\text { IV }\end{array}$ & $\begin{array}{l}\Delta \text { Invest } \\
\mathrm{V}\end{array}$ & $\begin{array}{l}\Delta \text { Revenue } \\
\text { VI }\end{array}$ \\
\hline Access to credit & $\begin{array}{l}1.900^{* *} \\
(0.86)\end{array}$ & $\begin{array}{l}1.904^{* *} \\
(0.93)\end{array}$ & $\begin{array}{l}3.786^{* * *} \\
(1.12)\end{array}$ & $\begin{array}{l}2.972^{* *} \\
(1.33)\end{array}$ & $\begin{array}{l}0.698^{* *} \\
(0.26)_{* * *}\end{array}$ & $\begin{array}{l}0.830^{* * *} \\
(0.28)_{* * *}\end{array}$ \\
\hline Prior constraint & $\begin{array}{l}0.098 \\
(0.22)\end{array}$ & $\begin{array}{l}0.136 \\
(0.24)\end{array}$ & $\begin{array}{l}-0.277 \\
(0.45)\end{array}$ & $\begin{array}{l}-0.144 \\
(0.42)\end{array}$ & $\begin{array}{l}0.357 \\
(0.10)_{* * *}\end{array}$ & $\begin{array}{l}0.938 \\
(0.27)\end{array}$ \\
\hline Initial output & $\begin{array}{l}0.085 \\
(0.11)_{* *}\end{array}$ & $\begin{array}{l}0.098 \\
(0.11)_{* *}\end{array}$ & $\begin{array}{l}0.167 \\
(0.27)_{*}\end{array}$ & $\begin{array}{l}0.114 \\
(0.15)\end{array}$ & $\begin{array}{l}0.130^{* * *} \\
(0.04)\end{array}$ & $\begin{array}{l}0.047 \\
(0.05)\end{array}$ \\
\hline $\begin{array}{l}\text { Initial country } \\
\text { income }\end{array}$ & $\begin{array}{l}1.350^{* *} \\
(0.54)\end{array}$ & $\begin{array}{l}1.238^{* *} \\
(0.49)\end{array}$ & $\begin{array}{l}2.339 \\
(1.21)\end{array}$ & $\begin{array}{l}1.850^{* *} \\
(0.76)\end{array}$ & $\begin{array}{l}-0.508^{*} \\
(0.26)\end{array}$ & $\begin{array}{l}0.143 \\
(0.31)\end{array}$ \\
\hline Year of privatization & $\begin{array}{l}0.145^{*} \\
(0.08)\end{array}$ & $\begin{array}{l}0.184^{* *} \\
(0.09)\end{array}$ & $\begin{array}{l}0.739 * * * \\
(0.23)\end{array}$ & $\begin{array}{l}0.231 * \\
(0.14)\end{array}$ & $\begin{array}{l}-0.079 * \\
(0.04)\end{array}$ & $\begin{array}{l}0.076 \\
(0.06)\end{array}$ \\
\hline $\begin{array}{l}\text { Independent } \\
\text { regulator }\end{array}$ & $\begin{array}{l}-0.315 \\
(0.92)\end{array}$ & $\begin{array}{l}0.798 \\
(0.67)\end{array}$ & $\begin{array}{l}-0.366 \\
(1.37)\end{array}$ & $\begin{array}{l}1.035 \\
(0.93)\end{array}$ & $\begin{array}{l}-0.634^{*} \\
(0.35)\end{array}$ & $\begin{array}{l}-1.435 \\
(0.90)\end{array}$ \\
\hline Competition & $\begin{array}{l}0.556^{* *} \\
(0.26)\end{array}$ & $\begin{array}{l}0.447 \\
(0.32)\end{array}$ & $\begin{array}{l}0.972 \\
(0.61)\end{array}$ & $\begin{array}{l}0.347 \\
(0.35)\end{array}$ & $\begin{array}{l}0.052 \\
(0.07)\end{array}$ & $\begin{array}{l}0.050 \\
(0.13)\end{array}$ \\
\hline Full privatization & & $\begin{array}{l}0.917 \\
(1.22)\end{array}$ & & & & \\
\hline French legal origin & & $\begin{array}{l}0.042 \\
(0.60)\end{array}$ & & & & \\
\hline Number of observations & 54 & 54 & 53 & 50 & 38 & 38 \\
\hline$R^{2}$ & 0.49 & 0.51 & 0.49 & 0.51 & 0.39 & 0.58 \\
\hline
\end{tabular}

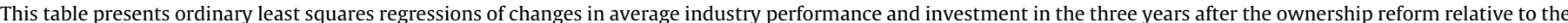

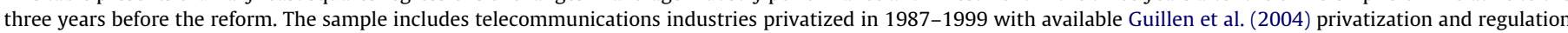

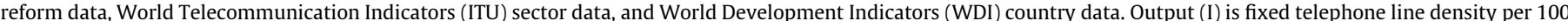

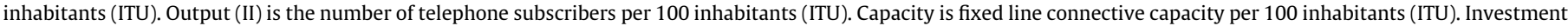

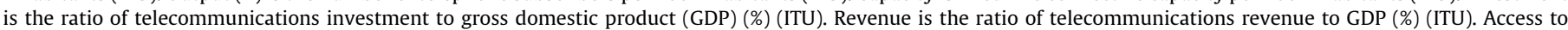

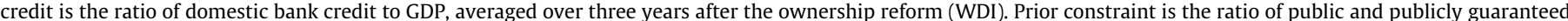

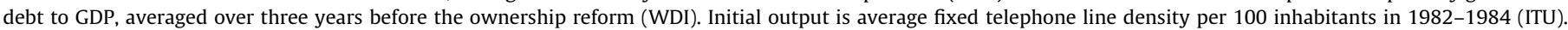

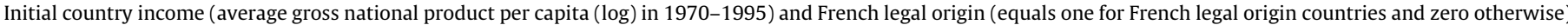

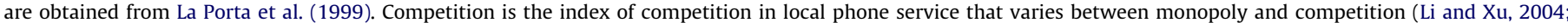

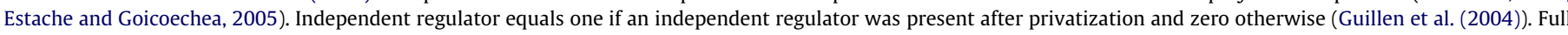

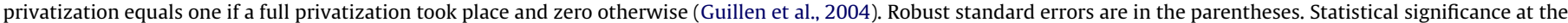
$1 \%, 5 \%$, and $10 \%$ level is denoted with ${ }^{* * *} * *$, and $*$, respectively. 
Table 4

Performance around ownership changes (sectoral study): Effects of equity market development, sovereign ratings, and reform characteristics.

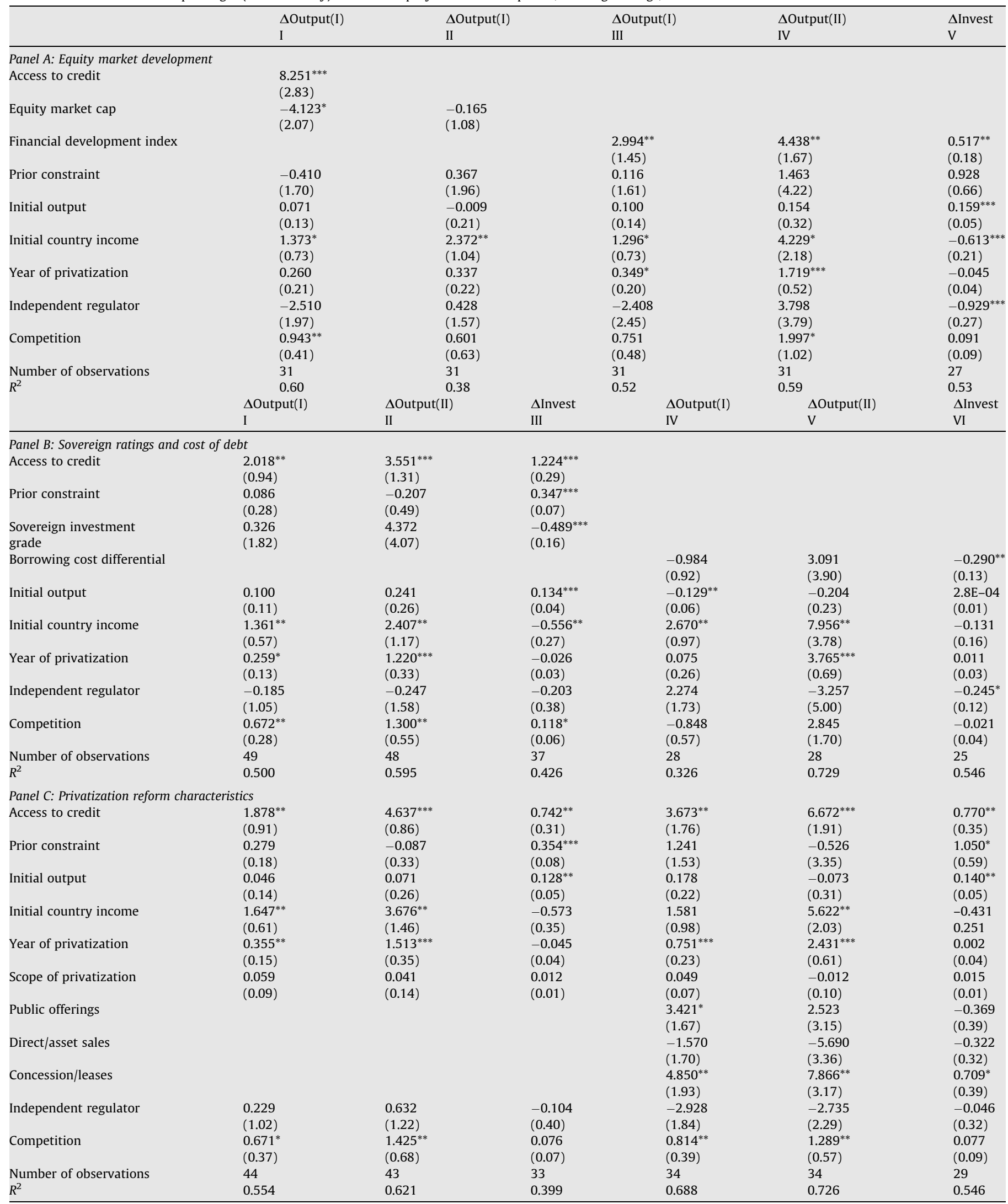

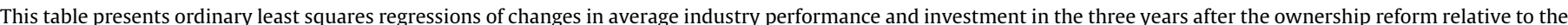

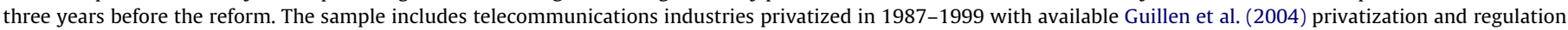

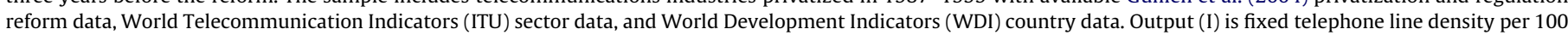

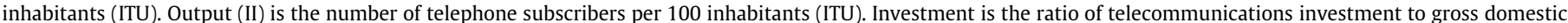

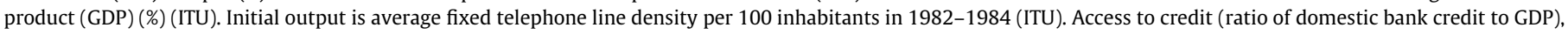




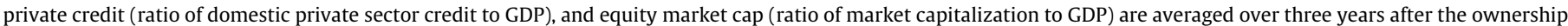

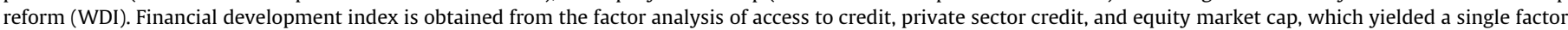

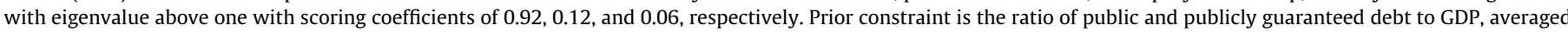

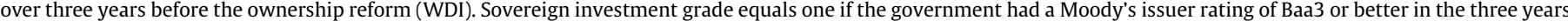

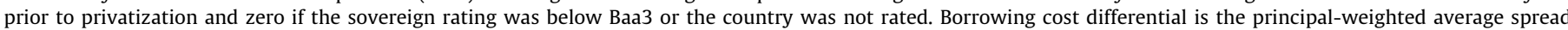

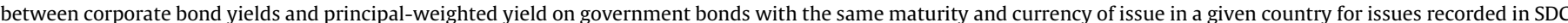

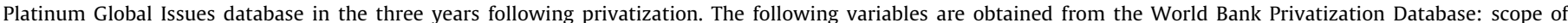

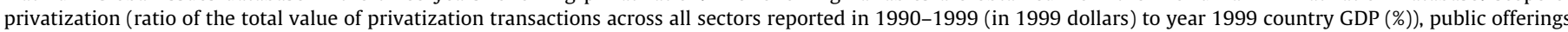

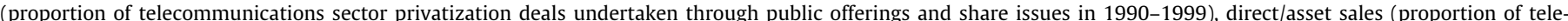

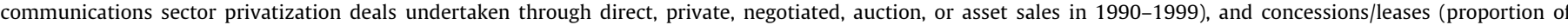

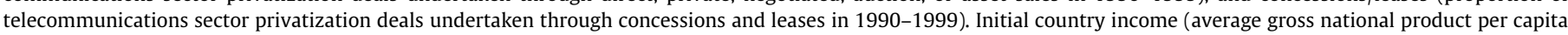

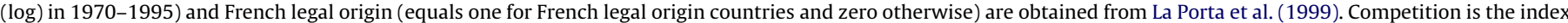

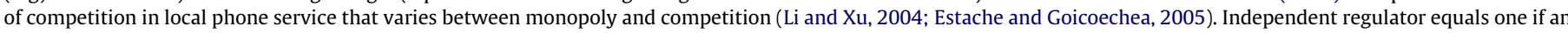

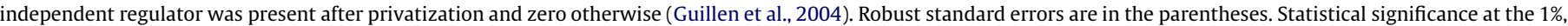
$5 \%$, and $10 \%$ level is denoted with $* * *, * *$, and $*$, respectively.

The following variables are used to explain ownership changes. Historical performance could affect the odds of privatization. Wellperforming companies can raise more capital as part of a privatization sale. Also, cash-strapped governments facing fiscal deficits could accelerate the sales of underperforming state-owned entities (Noll (2000)). Countries with the French legal tradition tend to have weaker shareholder rights (La Porta et al., 1998). As a result, governments in French legal origin countries could be more likely to retain concentrated stakes and defer the sale of state-owned entities to investors. Past population size and income are included to control for country-level heterogeneity. Following La Porta et al., 1999, we add two other proxies for exogenous country conditions: location (log of absolute value of latitude, with higher values corresponding to temperate climates, which could contribute to the formation of better institutions) and religious composition (percentage of Catholics as a proxy for social traditions and attitudes).

\section{Results}

Before turning to the analysis of performance changes, we examine the ownership reform decision. The ownership reform probability is predicted in Table 2 (columns I-III). As expected, industries in French legal origin countries are significantly less likely to be privatized. The sign of the coefficient on initial output is negative, which suggests that governments are more willing to privatize poorly performing enterprises. Historical average budget balance has a negative effect on the likelihood of a full privatization, implying that fiscally burdened governments are more likely to undertake a complete transfer of ownership rights. More developed countries favor privatizations with full transfer of ownership, all else given. Choice of the scope of ownership reforms (full, majority stake, minority stakes, none) is modeled using Ordered Probit and Multinomial Probit (Table 2, columns IV-V). The results are consistent with the evidence above. All else equal, a full privatization is more likely to occur in poorly performing industries; countries with fiscal deficits; countries with non-French legal origin; and larger, more developed economies.

We now turn to the analysis of performance after ownership reforms. In Table 3 we examine changes in performance and operating characteristics in the three years following privatization to identify the determinants of successful reforms and evaluate the relative importance of access to financing. The results in columns

Table 5

Performance around ownership changes (sectoral study): Selection model.

\begin{tabular}{|c|c|c|c|c|c|}
\hline & $\begin{array}{l}\Delta \text { Output(I) } \\
\text { I }\end{array}$ & $\begin{array}{l}\Delta \text { Output(II) } \\
\text { II }\end{array}$ & $\begin{array}{l}\Delta \text { Capacity } \\
\text { III }\end{array}$ & $\begin{array}{l}\Delta \text { Invest } \\
\text { IV }\end{array}$ & $\begin{array}{l}\Delta \text { Revenue } \\
\mathrm{V}\end{array}$ \\
\hline Access to credit & $\begin{array}{l}1.762^{* *} \\
(0.88)\end{array}$ & $\begin{array}{l}3.409^{* * *} \\
(1.08)\end{array}$ & $\begin{array}{l}2.776^{* *} \\
(1.24)\end{array}$ & $\begin{array}{l}0.641^{* * *} \\
(0.23)\end{array}$ & $\begin{array}{l}0.762^{* * *} \\
(0.28)\end{array}$ \\
\hline Prior constraint & $\begin{array}{c}0.144 \\
(0.22)\end{array}$ & $\begin{array}{c}-0.256 \\
(0.53)\end{array}$ & $\begin{array}{c}-0.164 \\
(0.34)\end{array}$ & $\begin{array}{l}0.362^{* * *} \\
(0.09)\end{array}$ & $\begin{array}{l}1.002^{* * *} \\
(0.25)\end{array}$ \\
\hline Initial output & $\begin{array}{c}0.112 \\
(0.10)\end{array}$ & $\begin{array}{r}0.273 \\
(0.25)\end{array}$ & $\begin{array}{r}0.176 \\
(0.14)\end{array}$ & $\begin{array}{l}0.135^{* * *} \\
(0.03)\end{array}$ & $\begin{array}{r}0.057 \\
(0.05)\end{array}$ \\
\hline Initial country income & $\begin{array}{l}1.235^{* * *} \\
(0.47)\end{array}$ & $\begin{array}{r}1.840 \\
(1.18)\end{array}$ & $\begin{array}{l}1.515^{* *} \\
(0.69)\end{array}$ & $\begin{array}{l}-0.540^{* *} \\
(0.24)\end{array}$ & $\begin{array}{c}0.102 \\
(0.31)\end{array}$ \\
\hline Year of privatization & $\begin{array}{c}0.119 \\
(0.08)\end{array}$ & $\begin{array}{l}0.640^{* * *} \\
(0.16)\end{array}$ & $\begin{array}{c}0.169 \\
(0.13)\end{array}$ & $\begin{array}{c}-0.084^{* *} \\
(0.04)\end{array}$ & $\begin{array}{c}0.072 \\
(0.05)\end{array}$ \\
\hline Independent regulator & $\begin{array}{c}0.889 \\
(0.73)\end{array}$ & $\begin{array}{c}0.195 \\
(1.40)\end{array}$ & $\begin{array}{r}0.962 \\
(0.93)\end{array}$ & $\begin{array}{c}-0.609^{*} \\
(0.36)\end{array}$ & $\begin{array}{c}-1.393^{*} \\
(0.72)\end{array}$ \\
\hline Competition & $\begin{array}{r}0.380 \\
(0.25)\end{array}$ & $\begin{array}{c}0.662 \\
(0.51)\end{array}$ & $\begin{array}{c}0.118 \\
(0.35)\end{array}$ & $\begin{array}{c}0.026 \\
(0.07)\end{array}$ & $\begin{array}{r}0.010 \\
(0.15)\end{array}$ \\
\hline Wald test & $4.76^{* *}$ & $7.73^{* * *}$ & $6.52^{* *}$ & 0.99 & 0.29 \\
\hline Number of observations & 54 & 53 & 50 & 38 & 38 \\
\hline
\end{tabular}

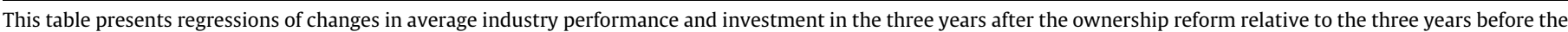

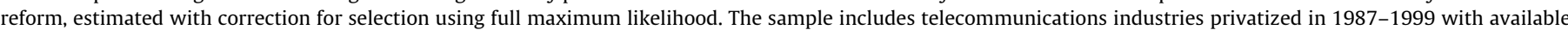

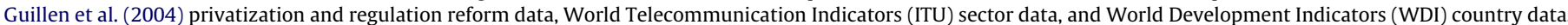

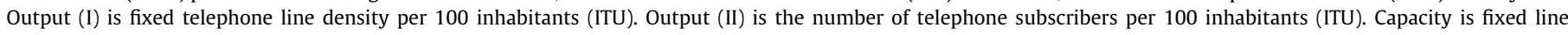

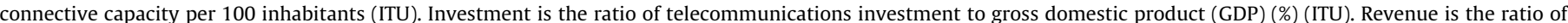

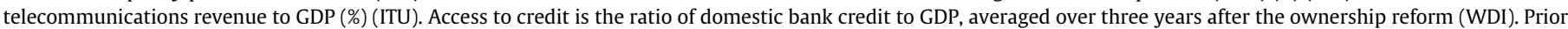

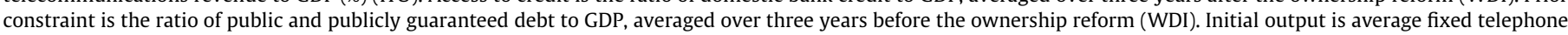

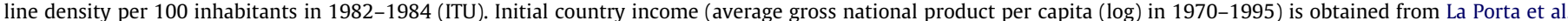

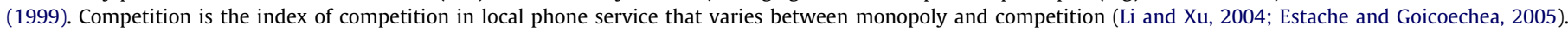

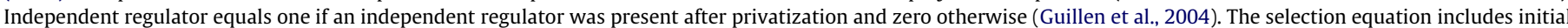

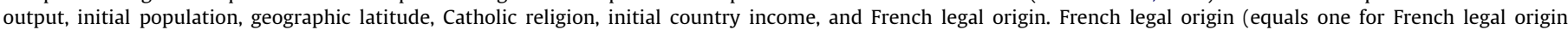

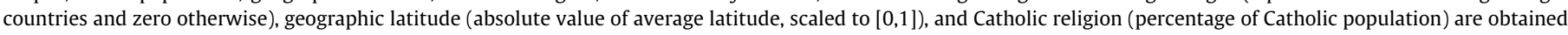

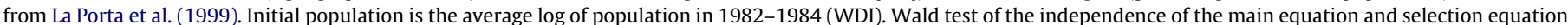
is reported. Robust standard errors are in the parentheses. Statistical significance at the $1 \%, 5 \%$, and $10 \%$ level is denoted with $* * * * *$, and *, respectively. 
Table 6

Performance around ownership changes (sectoral study): Fixed effects estimation.

\begin{tabular}{|c|c|c|c|c|c|}
\hline & $\begin{array}{l}\Delta \text { Output(I) } \\
\text { I }\end{array}$ & $\begin{array}{l}\Delta \text { Output(II) } \\
\text { II }\end{array}$ & $\begin{array}{l}\Delta \text { Capacity } \\
\text { III }\end{array}$ & $\begin{array}{l}\Delta \text { Invest } \\
\text { IV }\end{array}$ & $\begin{array}{l}\Delta \text { Revenue } \\
\mathrm{V}\end{array}$ \\
\hline Access to credit & $\begin{array}{l}3.486^{* * *} \\
(0.92)\end{array}$ & $\begin{array}{l}24.878^{* * *} \\
(4.12)\end{array}$ & $\begin{array}{l}5.399^{* *} \\
(2.74)\end{array}$ & $\begin{array}{l}0.432^{* * *} \\
(0.16)\end{array}$ & $\begin{array}{l}4.321^{* *} \\
(1.99)\end{array}$ \\
\hline$\Delta$ Income per capita & $\begin{array}{l}1.696^{* *} \\
(0.71)\end{array}$ & $\begin{array}{l}9.761^{* * *} \\
(3.37)\end{array}$ & $\begin{array}{l}1.195 \\
(1.22)\end{array}$ & $\begin{array}{l}0.207 \\
(0.16)\end{array}$ & $\begin{array}{l}1.958 \\
(1.46)\end{array}$ \\
\hline Years since privatization & $\begin{array}{l}0.847^{* * *} \\
(0.08)\end{array}$ & $\begin{array}{l}2.673^{* * *} \\
(0.26)\end{array}$ & $\begin{array}{l}0.935^{* * *} \\
(0.10)\end{array}$ & $\begin{array}{l}-0.027^{* * *} \\
(0.01)\end{array}$ & $\begin{array}{l}-0.182 \\
(0.14)\end{array}$ \\
\hline Independent regulator & $\begin{array}{l}0.617 \\
(0.56)\end{array}$ & $\begin{array}{l}18.724^{* * *} \\
(3.20)\end{array}$ & $\begin{array}{l}0.410 \\
(1.33)\end{array}$ & $\begin{array}{l}0.135^{*} \\
(0.08)\end{array}$ & $\begin{array}{l}-7.479^{* *} \\
(3.08)\end{array}$ \\
\hline Sector effects & Yes & Yes & Yes & Yes & Yes \\
\hline Year effects & Yes & Yes & Yes & Yes & Yes \\
\hline Number of observations & 629 & 601 & 319 & 388 & 423 \\
\hline
\end{tabular}

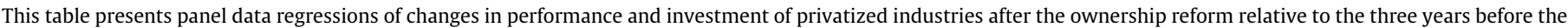

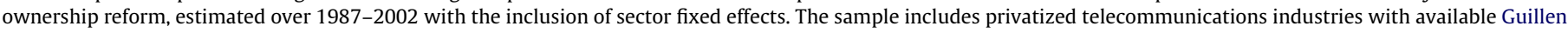

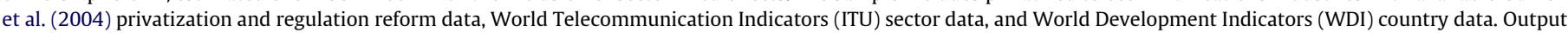

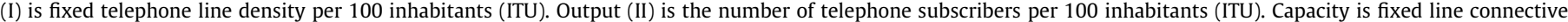

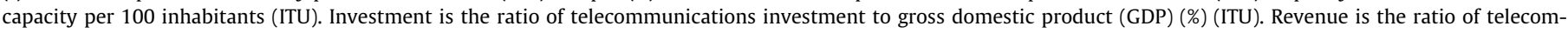

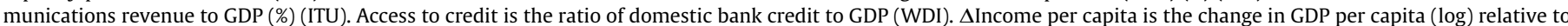

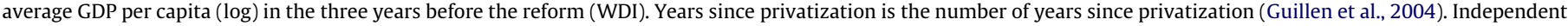

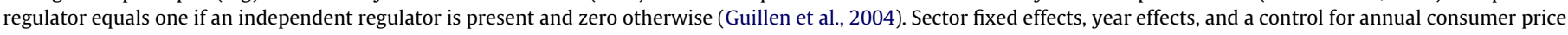

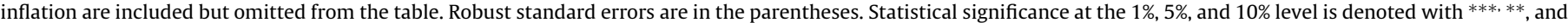
*, respectively.

I-IV and VI support the access to external financing hypothesis. Access to credit has a positive and significant effect on different measures of growth around ownership changes. Performance changes around ownership reforms are less sensitive to prior public sector constraints. The hypothesized positive effect of the prior public sector constraint is significant in the revenue specification (column $\mathrm{VI}$ ). Furthermore, privatizations undertaken in later years are associated with stronger performance improvements, consistent with learning about optimal privatization design.

We next examine changes in investment (column V). Access to credit has a positive effect on investment changes. Changes in investment of privatized companies do not appear to be driven by the elimination of soft budget constraints under the pressure of financial market discipline. Instead, recently privatized enterprises seem to be taking advantage of greater availability of external financing in environments with widespread access to credit. Further, industries subjected to more significant financing constraints under state ownership experience larger increases in investment after a change in ownership.

In addition to access to credit, equity market development could influence performance after privatization. Results of the analyses are presented in panel A of Table 4 . When market capitalization is included jointly with access to credit, it enters with a marginally significant coefficient while access to credit retains its positive sign and significance (column I). Multicollinearity due to a 0.74 correlation of market capitalization and access to credit is a potential concern. When included separately, however, market capitalization remains insignificant (column II). We also perform factor analysis of access to credit, private sector credit, and market capitalization measures. The resulting financial development index places most of the weight on access to credit and enters with a significant and positive coefficient (columns III-V). Newly privatized firms are likely to present significant information asymmetries to prospective investors. Because debt is less information-sensitive than equity, access to credit is a more significant determinant of performance of recently privatized sectors. We conclude that access to credit appears to have the greatest influence on performance, so we focus on this variable in subsequent analyses.

Tests of the prior constraint hypothesis reported in Table 3 rely on the extent of public sector indebtedness prior to privatization.
However, fiscally sound governments in countries with strong growth prospects could borrow extensively yet pay a relatively low cost of debt. As an alternative, we use the presence of an investment grade sovereign bond rating to capture the government's creditworthiness as a prospective borrower. The sovereign investment grade dummy equals one if Moody's sovereign issuer rating was Baa3 or above in the three years prior to privatization and zero if the rating was lower or if the country was not rated (Moody's Investors Service, 2003). If the prior constraint hypothesis holds, post-privatization gains would be stronger in countries that lacked a sovereign investment grade rating prior to the reform. Consistent with earlier findings, the prior constraint effect is significant for changes in investment but not for changes in performance, holding prior public debt levels constant (columns I-III of Table 4, panel B). The next robustness check evaluates the potential effects of a change from the public financing constraint to a private sector financing constraint after privatization. We use weighted average spreads between corporate and government bond yields in a given country to proxy for potential relaxation or tightening of borrowing constraints after the change from public to private ownership (SDC Global Issues). High positive spreads indicate that privatized enterprises likely face higher borrowing costs than sovereign issuers, which should have an adverse effect on investment and performance. We find a significant effect in the case of investment changes but not performance changes. The additional evidence is consistent with the findings in Table 3. Relaxation of the prior constraint only affects investment changes after ownership reforms.

Finally, we examine robustness of access to credit findings to the inclusion of reform characteristics. ${ }^{2}$ The results remain qualitatively similar after the inclusion of the cumulative volume of privatization transactions in the country and dummies for the use of public offerings, direct or asset sales, and concessions or leases in privatization reforms (Table 4, panel C).

The results of performance and investment regressions with correction for selection bias are reported in Table 5. The model of

\footnotetext{
2 In an unreported test, in addition to including the year of the telecommunications sector privatization, we control for the number of years between the first privatization transaction in the World Bank Privatization Database and the telecommunications sector privatization. The results are qualitatively similar.
} 
Table 7

Long-term changes in performance (sectoral study): Full sample.

\begin{tabular}{|c|c|c|c|c|c|c|c|c|}
\hline & $\begin{array}{l}\Delta \text { Output(I) } \\
\text { I }\end{array}$ & $\begin{array}{l}\Delta \text { Output(I) } \\
\text { II }\end{array}$ & $\begin{array}{l}\Delta \text { Output(I) } \\
\text { III }\end{array}$ & $\begin{array}{l}\Delta \text { Output(I) } \\
\text { IV }\end{array}$ & $\begin{array}{l}\Delta \text { Output(II) } \\
\mathrm{V}\end{array}$ & $\begin{array}{l}\Delta \text { Output(II) } \\
\text { VI }\end{array}$ & $\begin{array}{l}\Delta \text { Output(I) } \\
\text { VII }\end{array}$ & $\begin{array}{l}\Delta \text { Output(II) } \\
\text { VIII }\end{array}$ \\
\hline \multicolumn{9}{|c|}{ Panel A: Long-term changes in performance } \\
\hline Access to credit 1987-1999 & $\begin{array}{l}3.785^{* * *} \\
(1.37)\end{array}$ & & & & $\begin{array}{l}3.909^{* * *} \\
(1.36)\end{array}$ & & $\begin{array}{l}3.745^{* * *} \\
(1.37)\end{array}$ & $\begin{array}{l}3.904^{* * *} \\
(1.37)\end{array}$ \\
\hline Access to credit $1990-1999$ & & $\begin{array}{l}3.772^{* * *} \\
(1.40)\end{array}$ & & & & & & \\
\hline Access to credit 1987-1989 & & & $\begin{array}{l}3.659^{* * *} \\
(1.38)\end{array}$ & & & & & \\
\hline Private credit 1987-1999 & & & & $\begin{array}{l}4.389 * * \\
(2.20)\end{array}$ & & $\begin{array}{l}4.454^{* *} \\
(2.19)\end{array}$ & & \\
\hline Prior constraint & $\begin{array}{c}-0.681 \\
(1.10)\end{array}$ & $\begin{array}{c}-1.577 \\
(1.10)\end{array}$ & $\begin{array}{c}-1.667 \\
(1.12)\end{array}$ & $\begin{array}{c}-0.634 \\
(1.08)\end{array}$ & $\begin{array}{c}-1.434 \\
(1.10)\end{array}$ & $\begin{array}{c}-0.356 \\
(1.08)\end{array}$ & $\begin{array}{c}-1.675 \\
(1.09)\end{array}$ & $\begin{array}{c}-1.436 \\
(1.09)\end{array}$ \\
\hline Privatization & $\begin{array}{c}0.564 \\
(2.72)\end{array}$ & $\begin{array}{r}0.560 \\
(2.72)\end{array}$ & $\begin{array}{r}1.677 \\
(2.68)\end{array}$ & $\begin{array}{r}1.363 \\
(2.78)\end{array}$ & $\begin{array}{r}0.677 \\
(2.70)\end{array}$ & $\begin{array}{r}1.497 \\
(2.77)\end{array}$ & & \\
\hline Full privatization & & & & & & & $\begin{array}{c}1.150 \\
(2.62)\end{array}$ & $\begin{array}{c}1.066 \\
(2.61)\end{array}$ \\
\hline Initial output & $\begin{array}{l}0.854^{* * *} \\
(0.30)\end{array}$ & $\begin{array}{l}0.848^{* * *} \\
(0.30)\end{array}$ & $\begin{array}{l}0.742^{* *} \\
(0.31)\end{array}$ & $\begin{array}{l}0.802^{* * *} \\
(0.30)\end{array}$ & $\begin{array}{l}0.848^{* * *} \\
(0.29)\end{array}$ & $\begin{array}{l}0.795^{* * *} \\
(0.30)\end{array}$ & $\begin{array}{l}0.860^{* * *} \\
(0.28)\end{array}$ & $\begin{array}{l}0.858^{* * *} \\
(0.28)\end{array}$ \\
\hline Initial country income & $\begin{array}{l}1.789^{* *} \\
(0.80)\end{array}$ & $\begin{array}{l}1.842^{* *} \\
(0.80)\end{array}$ & $\begin{array}{l}1.887^{* *} \\
(0.83)\end{array}$ & $\begin{array}{l}1.912^{* *} \\
(0.82)\end{array}$ & $\begin{array}{l}1.816^{* *} \\
(0.80)\end{array}$ & $\begin{array}{l}1.949^{* *} \\
(0.81)\end{array}$ & $\begin{array}{l}1.692^{* *} \\
(0.80)\end{array}$ & $\begin{array}{l}1.727^{* *} \\
(0.80)\end{array}$ \\
\hline Indebted & $\begin{array}{c}-0.489 \\
(1.09)\end{array}$ & $\begin{array}{c}-0.451 \\
(1.09)\end{array}$ & $\begin{array}{c}-0.771 \\
(1.14)\end{array}$ & $\begin{array}{c}-0.754 \\
(1.11)\end{array}$ & $\begin{array}{c}-0.623 \\
(1.08)\end{array}$ & $\begin{array}{c}-0.895 \\
(1.11)\end{array}$ & $\begin{array}{c}-0.572 \\
(1.08)\end{array}$ & $\begin{array}{c}-0.689 \\
(1.08)\end{array}$ \\
\hline Independent regulator & $\begin{array}{c}-0.039 \\
(1.07)\end{array}$ & $\begin{array}{c}-0.007 \\
(1.07)\end{array}$ & $\begin{array}{c}-0.057 \\
(1.12)\end{array}$ & $\begin{array}{c}-0.126 \\
(1.09)\end{array}$ & $\begin{array}{c}-0.059 \\
(1.06)\end{array}$ & $\begin{array}{c}-0.151 \\
(1.08)\end{array}$ & $\begin{array}{c}-0.141 \\
(1.00)\end{array}$ & $\begin{array}{r}-0.107 \\
(0.99)\end{array}$ \\
\hline Competition & $\begin{array}{c}0.291 \\
(0.52)\end{array}$ & $\begin{array}{r}0.291 \\
(0.52)\end{array}$ & $\begin{array}{c}0.040 \\
(0.54)\end{array}$ & $\begin{array}{c}0.140 \\
(0.53)\end{array}$ & $\begin{array}{c}0.293 \\
(0.51)\end{array}$ & $\begin{array}{c}0.140 \\
(0.53)\end{array}$ & $\begin{array}{r}0.232 \\
(0.53)\end{array}$ & $\begin{array}{c}0.248 \\
(0.52)\end{array}$ \\
\hline \multirow[t]{2}{*}{ Number of observations } & \multicolumn{2}{|c|}{$\begin{array}{l}\text { Access to credit 1987- } \\
1999\end{array}$} & \multicolumn{2}{|c|}{ Private credit 1987-1999 } & \multicolumn{2}{|c|}{ Prior constraint } & \multicolumn{2}{|c|}{ French legal origin } \\
\hline & $\begin{array}{l}\text { High } \\
\text { I }\end{array}$ & $\begin{array}{l}\text { Low } \\
\text { II }\end{array}$ & $\begin{array}{l}\text { High } \\
\text { III }\end{array}$ & $\begin{array}{l}\text { Low } \\
\text { IV }\end{array}$ & $\begin{array}{l}\text { High } \\
\mathrm{V}\end{array}$ & $\begin{array}{l}\text { Low } \\
\text { VI }\end{array}$ & $\begin{array}{l}\text { Yes } \\
\text { VII }\end{array}$ & $\begin{array}{l}\text { No } \\
\text { VIII }\end{array}$ \\
\hline \multicolumn{9}{|c|}{ Panel B: Long-term changes in performance (subsamples) } \\
\hline $\begin{array}{l}\text { Effect of privatization on } \\
\Delta \text { Output(I) }\end{array}$ & $\begin{array}{r}2.253 \\
(3.92)\end{array}$ & $\begin{array}{c}0.232 \\
(3.26)\end{array}$ & $\begin{array}{c}1.694 \\
(3.69)\end{array}$ & $\begin{array}{c}-0.366 \\
(2.64)\end{array}$ & $\begin{array}{r}3.035 \\
(3.85)\end{array}$ & $\begin{array}{l}2.484 \\
(2.90)\end{array}$ & $\begin{array}{c}-0.837 \\
(2.76)\end{array}$ & $\begin{array}{c}-1.292 \\
(4.93)\end{array}$ \\
\hline Effect of privatization on & 2.084 & 0.384 & 1.668 & -0.697 & 3.282 & 2.207 & -0.915 & -1.211 \\
\hline$\Delta$ Output(II) & $(3.93)$ & $(3.18)$ & $(3.69)$ & $(2.50)$ & $(3.81)$ & $(2.89)$ & $(2.73)$ & $(4.85)$ \\
\hline Number of observations & 43 & 45 & 44 & 44 & 44 & 45 & 53 & 36 \\
\hline
\end{tabular}

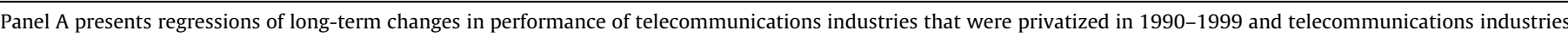

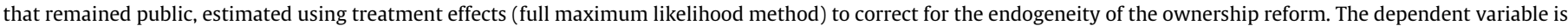

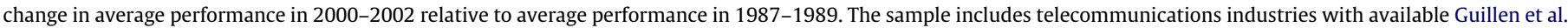

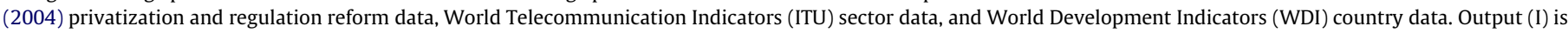

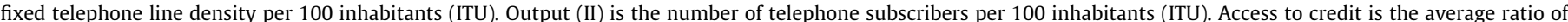

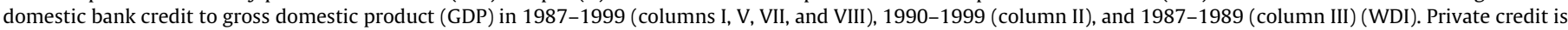

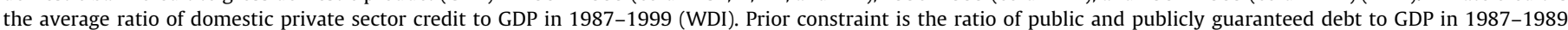

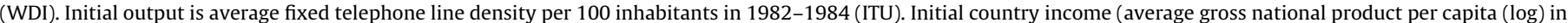

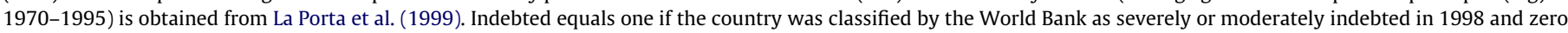

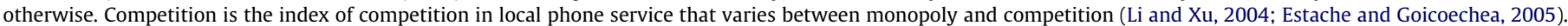
Independent regulator equals one if an independent regulator was present and zero otherwise (Guillen et al. (2004)).

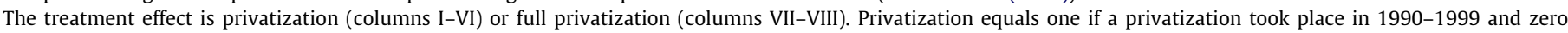

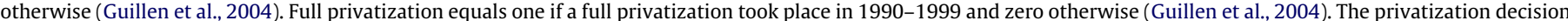

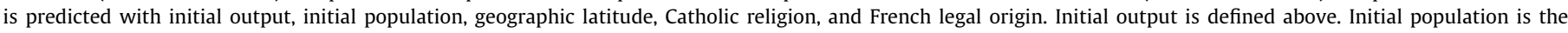

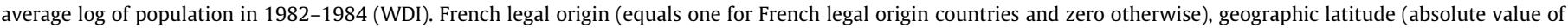
average latitude, scaled to [0,1]), and Catholic religion (percentage of Catholic population) are obtained from La Porta et al. (1999).

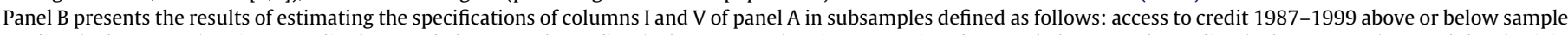

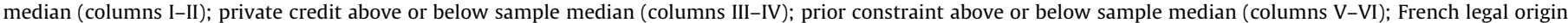
equals one or zero (columns VII-VIII). Variables used to define subsamples are excluded from respective specifications.

Robust standard errors are in the parentheses. Statistical significance at the $1 \%, 5 \%$, and $10 \%$ level is denoted with $* * * * *$, and $*$, respectively.

the ownership change decision is adopted from column I of Table 2. Similar to previous tables, we consider changes in performance in the three years after the ownership reform compared with average performance in the three years before the reform.

The signs of coefficients of interest are consistent with previously presented ordinary least squares evidence. Access to credit has a positive and significant effect on performance and investment changes. Gains in performance and investment from better access to credit are generated already in the first three years after the ownership change. Investment changes remain positively correlated with prior constraint, indicating that previously constrained industries increase investment at a faster rate after the switch to private ownership.
Tests presented in the previous tables use differences in average performance before and after the ownership change, resulting in cross-sectional regressions with one observation per sector. We depart from this variable construction approach in Table 6, which presents fixed effects estimation results using an unbalanced panel of changes in performance and investment following ownership reforms. Fixed effects estimation partly mitigates selection bias by controlling for unobservable time-invariant cross-sectional heterogeneity associated with the likelihood of privatization. Dependent variables are adjusted for the pre-privatization average but are allowed to vary over time. Due to the inclusion of fixed effects, time-invariant measures such as prior constraint are omitted. In all specifications, access to credit facilitates improvements 
Table 8

Performance around ownership changes (sectoral study): Selection model robustness checks.

\begin{tabular}{|c|c|c|c|c|c|c|}
\hline & $\begin{array}{l}\Delta \text { Output(I) } \\
\text { I }\end{array}$ & $\begin{array}{l}\Delta \text { Output(I) } \\
\text { II }\end{array}$ & $\begin{array}{l}\Delta \text { Output(II) } \\
\text { III }\end{array}$ & $\begin{array}{l}\Delta \text { Output(II) } \\
\text { IV }\end{array}$ & $\begin{array}{l}\Delta \text { Invest } \\
\mathrm{V}\end{array}$ & $\begin{array}{l}\Delta \text { Invest } \\
\text { VI }\end{array}$ \\
\hline Access to credit & & $\begin{array}{l}1.940 * * \\
(0.80)\end{array}$ & & $\begin{array}{l}3.525^{* * *} \\
(1.05)\end{array}$ & & $\begin{array}{l}0.664^{* *} \\
(0.29)\end{array}$ \\
\hline Private credit & $\begin{array}{l}2.377^{* *} \\
(1.21)\end{array}$ & & $\begin{array}{l}5.327^{* * *} \\
(1.94)\end{array}$ & & $\begin{array}{l}0.589 * * \\
(0.25)\end{array}$ & \\
\hline Prior constraint & $\begin{array}{l}0.596^{* *} \\
(0.295)\end{array}$ & & $\begin{array}{c}0.670 \\
(0.85)\end{array}$ & & $\begin{array}{l}0.441^{* *} \\
(0.179)\end{array}$ & \\
\hline $\begin{array}{l}\text { Prior external debt } \\
\text { constraint }\end{array}$ & & $\begin{array}{c}-0.003 \\
(0.196)\end{array}$ & & $\begin{array}{r}-0.281 \\
(0.50)\end{array}$ & & $\begin{array}{l}0.388^{* * *} \\
(0.134)\end{array}$ \\
\hline Initial output & $\begin{array}{r}0.017 \\
(0.14)\end{array}$ & $\begin{array}{c}0.141 \\
(0.10)\end{array}$ & $\begin{array}{r}0.133 \\
(0.34)\end{array}$ & $\begin{array}{r}0.271 \\
(0.25)\end{array}$ & $\begin{array}{l}0.149^{* * *} \\
(0.05)\end{array}$ & $\begin{array}{l}0.126^{* * *} \\
(0.04)\end{array}$ \\
\hline Initial country income & $\begin{array}{l}1.238^{* *} \\
(0.52)\end{array}$ & $\begin{array}{l}1.054^{* *} \\
(0.46)\end{array}$ & $\begin{array}{c}1.824 \\
(1.14)\end{array}$ & $\begin{array}{r}1.811 \\
(1.15)\end{array}$ & $\begin{array}{l}-0.480^{* *} \\
(0.22)\end{array}$ & $\begin{array}{c}-0.496^{*} \\
(0.27)\end{array}$ \\
\hline Year of privatization & $\begin{array}{c}0.054 \\
(0.08)\end{array}$ & $\begin{array}{c}0.108 \\
(0.07)\end{array}$ & $\begin{array}{l}0.551^{* * *} \\
(0.16)\end{array}$ & $\begin{array}{l}0.645^{* * *} \\
(0.16)\end{array}$ & $\begin{array}{c}-0.072^{*} \\
(0.04)\end{array}$ & $\begin{array}{c}-0.075^{*} \\
(0.04)\end{array}$ \\
\hline Independent regulator & & $\begin{array}{c}-0.356 \\
(0.85)\end{array}$ & & $\begin{array}{r}0.181 \\
(1.21)\end{array}$ & & $\begin{array}{c}-0.086 \\
(0.37)\end{array}$ \\
\hline $\begin{array}{l}\text { Semi-autonomous } \\
\text { regulator }\end{array}$ & $\begin{array}{c}-1.037 \\
(0.83)\end{array}$ & & $\begin{array}{r}-1.607 \\
(1.47)\end{array}$ & & $\begin{array}{c}-0.442 \\
(0.29)\end{array}$ & \\
\hline Competition & $\begin{array}{r}0.413 \\
(0.26)\end{array}$ & $\begin{array}{l}0.487^{* *} \\
(0.25)\end{array}$ & $\begin{array}{r}0.619 \\
(0.58)\end{array}$ & $\begin{array}{r}0.753 \\
(0.52)\end{array}$ & $\begin{array}{r}0.010 \\
(0.06)\end{array}$ & $\begin{array}{r}0.031 \\
(0.07)\end{array}$ \\
\hline Geographic latitude & $\begin{array}{c}4.254 \\
(3.59)\end{array}$ & & $\begin{array}{r}6.323 \\
(8.23)\end{array}$ & & $\begin{array}{c}-0.726 \\
(0.52)\end{array}$ & \\
\hline Geographic area & $\begin{array}{r}-0.133 \\
(0.14)\end{array}$ & $\begin{array}{c}-0.135 \\
(0.10)\end{array}$ & $\begin{array}{c}-0.138 \\
(0.36)\end{array}$ & $\begin{array}{c}-0.175 \\
(0.26)\end{array}$ & $\begin{array}{c}-0.014 \\
(0.05)\end{array}$ & $\begin{array}{r}-0.023 \\
(0.05)\end{array}$ \\
\hline Wald test & $7.64^{* * *}$ & $3.91^{* *}$ & $9.1^{* * *}$ & $7.56^{* * *}$ & $2.83^{*}$ & 1.31 \\
\hline Number of observations & 54 & 54 & 53 & 53 & 38 & 38 \\
\hline
\end{tabular}

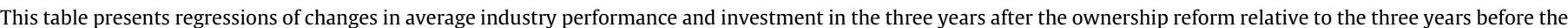

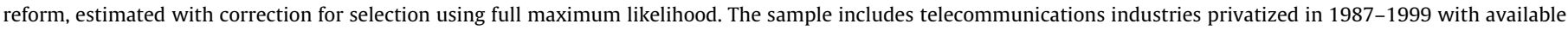

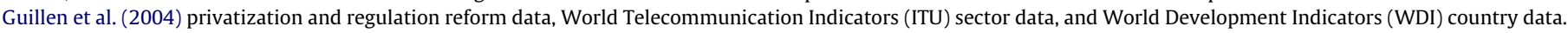

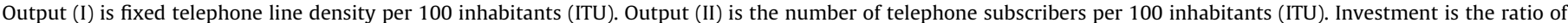

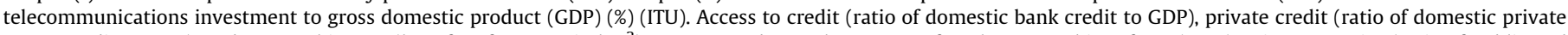

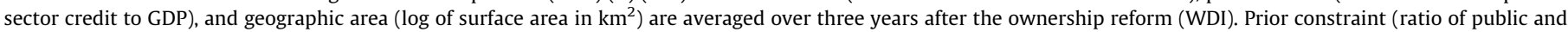

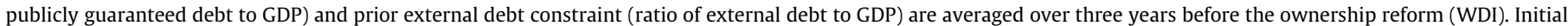

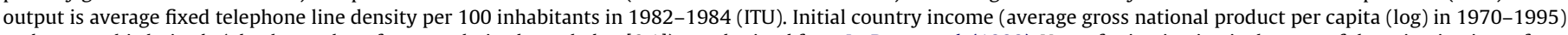

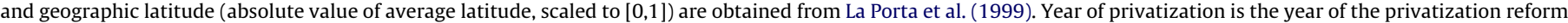

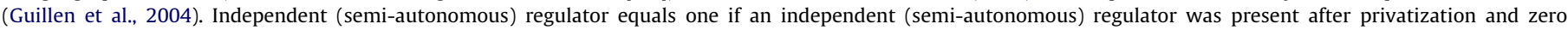

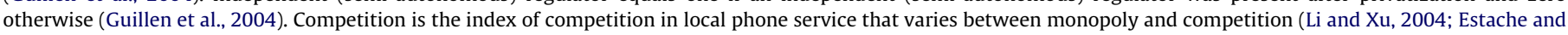

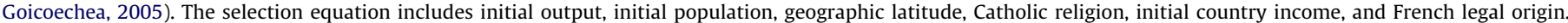

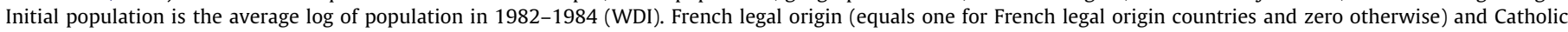

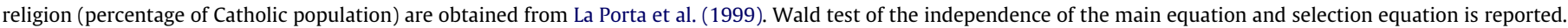
Robust standard errors are in the parentheses. Statistical significance at the $1 \%, 5 \%$, and $10 \%$ level is denoted with $* * *, * *$, and *, respectively.

in performance and growth in investment, consistent with the findings in the previous tables.

Next, we analyze the effects of privatization and access to financing on long-term changes in performance in the full sample that includes both privatized and publicly owned industries (Table 7 ). Access to credit, measured over different time periods (19871999, 1990-1999, and 1987-1989), has a positive effect on longterm performance changes, which is consistent with the role of access to credit for performance improvements among privatized industries. Industries in severely indebted countries experience setbacks in performance. This full sample effect is not surprising: Publicly owned industries suffer from the government's limited ability to borrow, whereas the effect of prior constraint on the performance of privatized industries was not found to be significant. Initial output and country income are positively associated with subsequent growth in output.

Coefficients on the privatization and full privatization variables are not statistically significant. Although ownership reforms are accompanied by significant performance increases, the ownership change sample on average does not outperform the sample of publicly owned industries, all else equal. Our results lend support to the view that the cost of ownership reforms should be traded off against performance gains due to strengthened incentives and other improvements. The substitution of guaranteed government financing for the need to raise financing from banks and private investors (initially without a strong reputation in external capital markets) could prevent privatized entities from fully capitalizing on improvements in incentives. Costs of private financing also are higher if the risk of privatization reversal (future nationalization of the privatized company) is not trivial. Finally, previously unconstrained firms could experience little improvement in access to capital after privatization.

To identify industries that were most affected by ownership changes, we divide the full sample into subsamples based on access to credit, prior constraints, and legal origin (panel B of Table 7). Industries that enjoy better credit access after privatization are expected to benefit more from the ownership change, and vice versa. However, the privatization effect remains insignificant across the different subsamples. ${ }^{3}$ Further, institutional barriers to shareholder rights as well as potential risks to property rights (such as privatization reversals) are expected to be higher in French legal origin environments (La Porta et al., 1998). Boubakri et al. (2009) find that the negative effect of government ownership is mitigated by the presence of sound institutions. The effect of ownership reforms on performance is insignificant in both subsamples. A similar pattern is observed in the subsamples based on prior constraint.

Further robustness checks verify the sensitivity of the main performance results to alternative definitions of the main variables and the inclusion of additional controls (Table 8).

\footnotetext{
${ }^{3}$ In an unreported test, we exclude countries with investment-grade ratings in 1987-1989 from the full sample, but the privatization effect remains insignificant.
} 
Table 9

Performance around ownership changes (multi-sector study): Fixed effects estimation on firm-level data.

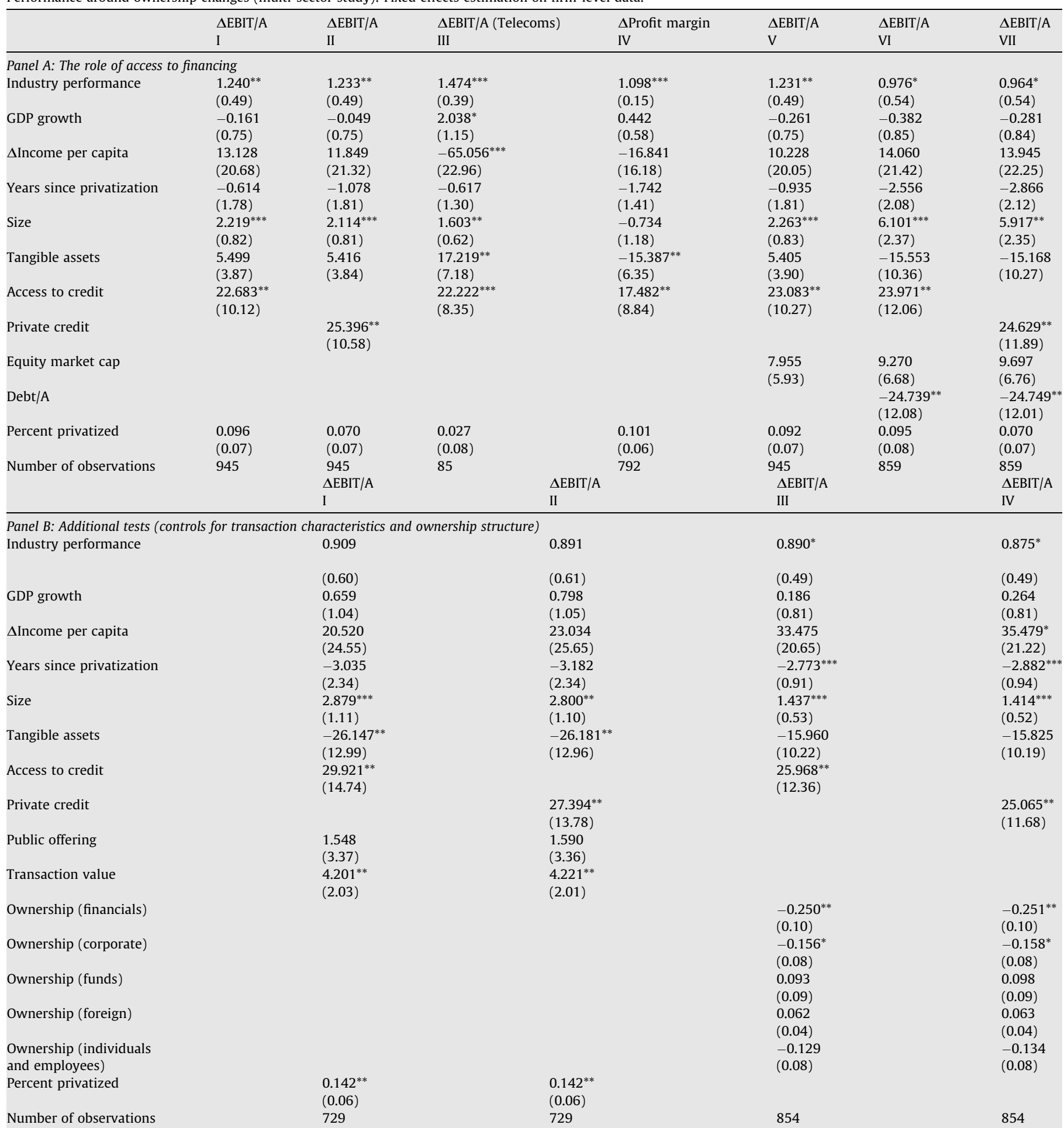

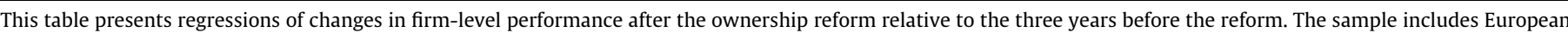

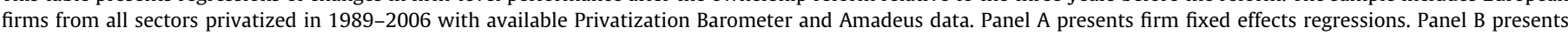

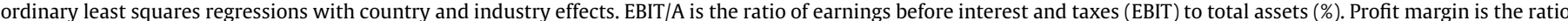

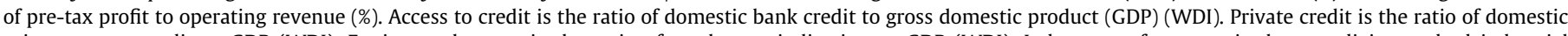

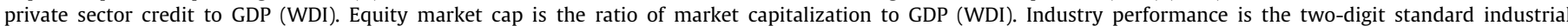

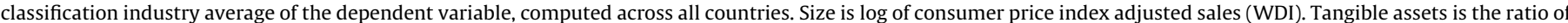

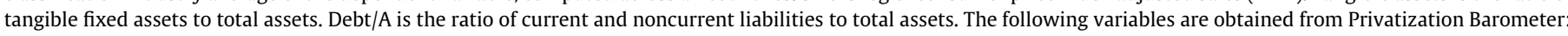

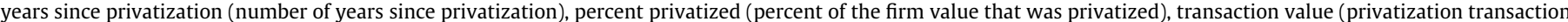

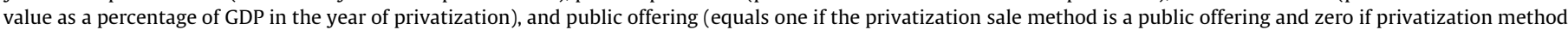

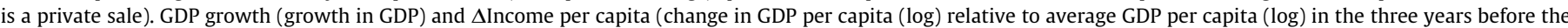

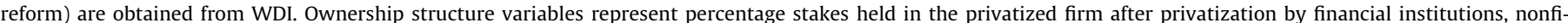

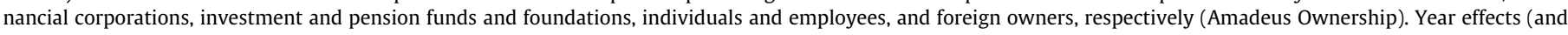

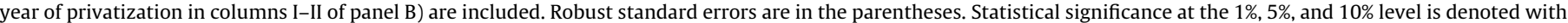
$* * *, * *$, and *, respectively. 
While the emphasis is on bank credit, we conduct a robustness check using private sector credit. Private sector credit enters with a significant positive coefficient, as expected. An alternative measure of prior public sector constraint, ratio of external debt to GDP, enters significantly in the investment growth regression. Previously constrained industries exhibit significantly higher investment growth after privatization.

The results presented so far were based on telecommunications sector data. While this research design has clear advantages, the last set of tests extends the sample to incorporate all sectors. We also refine the definitions of the dependent variables to focus on firm-level measures of profitability, which could offer another dimension of performance not captured in the preceding analysis. Firm-level results are reported in Table 9. Firm fixed effects and year effects are included. Profitability measures are adjusted for average profitability in the three years prior to the first reported ownership change.

Access to credit contributes to higher profitability. A $10 \%$ increase in bank credit as a share of GDP is associated with $2.3 \%$ higher profitability changes among privatized companies. The effect continues to hold when the sample is restricted to telecommunications companies and when the dependent variable is replaced with the change in the profit margin. The privatized percentage stake does not enter significantly, consistent with the evidence presented in Table 7. Other controls, such as industry performance and size, enter with expected signs.

In columns V-VII of panel A, we account for the role of equity financing for firm-level performance. As in sector-level analyses, access to credit retains its significant positive effect but market capitalization is not significant. In addition, we control for leverage defined as the ratio of total liabilities to total assets (columns VIVII). Although this variable could be endogenous, there is a positive association between firm performance and reliance on equity capital, consistent with prior work (Lang et al., 1996).

The use of firm fixed effects in panel A of Table 9 controls for time-invariant firm-level heterogeneity. Therefore, time-invariant controls are omitted. In panel B of Table 9, firm fixed effects are replaced with country and industry fixed effects, and time-invariant privatization transaction characteristics (the value sold as a percentage of GDP and privatization sale method, identified as public offering or private sale in Privatization Barometer) are introduced. Higher transaction values are associated with better subsequent performance. After the introduction of the controls, the privatized stake coefficient becomes significant, which indicates that the performance of privatized companies is increasing in the stake sold although privatization itself is not associated with better performance (columns I-II). Further, access to credit results continue to hold after the inclusion of controls for post-privatization ownership structure. Stakes by financial institutions and nonfinancial corporate owners enter with negative coefficients. Foreign ownership has a positive effect, and ownership by individuals and employees has a negative effect, although both coefficients are not statistically significant (columns III-IV).

\section{Conclusion}

The performance and growth implications of access to financing are examined in the context of major ownership changes. This paper offers new evidence on the effects of financing constraints, access to credit, and the performance-ownership relation around large changes in ownership in the course of ownership reforms. Our hypotheses address two issues: the impact of access to credit on performance and investment decisions following ownership changes and the implications of borrowing constraints faced by the public sector prior to the change in ownership for subsequent growth. The main findings are as follows.
First, access to credit facilitates performance improvements and investment growth following ownership reforms. Bank lending in particular is key to growth in investment, capacity, output, and profitability among recently privatized enterprises. Second, prior public sector financing constraint has a significant positive effect on investment around ownership changes. Previously constrained industries grow faster following a move to private ownership and relaxation of financing restrictions than their unconstrained counterparts. Third, the ownership reform sample fails to outperform the sample of publicly owned industries. The effect of ownership reforms remains insignificant also when conditions such as financial development, legal environment, and prior borrowing constraints are taken into account.

Our empirical analysis employs a comprehensive international telecommunications sector data set, which offers several advantages: broad country representation, relevance of access to financing due to capital intensity of the chosen industry, and homogeneity in the measurement of operating characteristics. Further, compared with aggregate economic outcomes, growth and performance changes in a single sector are less likely to drive country-level credit development, mitigating reverse causality concerns. A second set of tests uses a multi-sector sample of European privatizations to examine the effects of access to credit on firm performance around ownership changes. The empirical methodology accounts for the endogeneity of ownership reforms using a privatization decision model as well as fixed effects.

\section{Acknowledgements}

We thank the editor (Ike Mathur) and an anonymous referee for helpful suggestions. We are grateful to Bruce Greenwald, Kose John, Daniel Wolfenzon, David Yermack, and Bernard Yeung for valuable discussions. We thank Meghana Ayyagari, Sergey Barabanov, Nicholas Economides, William Greene, Madhu Kalimipalli, Frederic Sterbenz, and participants at the Sixth INFINITI Conference on International Finance, 2007 Financial Management Association meeting, 2007 Southwestern Finance Association meeting, and 2006 North American Productivity Workshop IV for helpful comments; Mauro Guillen, Witold Henisz, and Bennet Zellner for providing privatization and regulation data; Wei Li and Lixin Colin Xu for providing competition data; and Suzanne Bell for data assistance. Part of this work was undertaken while Anzhela Knyazeva and Diana Knyazeva were at the Leonard N. Stern School of Business, New York University. All remaining errors and omissions are ours.

\section{References}

Beck, T., Demirguc-Kunt, A., Maksimovic, V., 2006. The influence of financial and legal institutions on firm size. Journal of Banking and Finance 30, 2995-3015.

Beck, T., Levine, R., 2002. Industry growth and capital allocation: Does having a market- or bank-based system matter? Journal of Financial Economics 64, 147180.

Bortolotti, B., De Jong, F., Nicodano, G., Schindele, I., 2007. Privatization and stock market liquidity. Journal of Banking and Finance 31, 297-316.

Bortolotti, B., D'Souza, J., Fantini, M., Megginson, W., 2002. Privatization and the sources of performance improvement in the global telecommunications industry. Telecommunications Policy 26, 243-268.

Boubakri, N., Cosset, J.-C., 1998. The financial and operating performance of newly privatized firms: Evidence from developing countries. Journal of Finance 53, 1081-1110.

Boubakri, N., Cosset, J.-C., Guedhami, O., 2005. Liberalization, corporate governance and the performance of privatized firms in developing countries. Journal of Corporate Finance 11, 767-790.

Boubakri, N., Cosset, J.-C., Guedhami, O., 2009. From state to private ownership: Issues from strategic industries. Journal of Banking and Finance 33, 367-379.

Demirguc-Kunt, A., Maksimovic, V., 1998. Law, finance, and firm growth. Journal of Finance 53, 2107-2137.

Dewenter, K., Malatesta, P., 2001. State-owned and privately-owned firms: An empirical analysis of profitability, leverage, and labor intensity. American Economic Review 91, 320-334. 
D’Souza, J., Megginson, W., 1999. The financial and operating performance of privatized firms during the 1990s. Journal of Finance 54, 1397-1438.

D’Souza, J., Megginson, W., Nash, R., 2001. Determinants of performance improvements in privatized firms: The role of restructuring and corporate governance. Working paper, University of Oklahoma.

Estache, A., Goicoechea, A., 2005. A 'research' database on infrastructure economic performance. Working Paper, World Bank.

Frydman, R., Gray, C., Hessel, M., Rapaczynski, A., 1999. When does privatization work? The impact of private ownership on corporate performance in the transition economies. Quarterly Journal of Economics 114, 1153-1191.

Guillen, M., Henisz, W., Zelner, B., 2004. International coercion, emulation and policy diffusion: Market-oriented infrastructure reforms, 1977-1999. Working Paper, University of Pennsylvania.

Gupta, N., 2005. Partial privatization and firm performance. Journal of Finance 60, 987-1015

Hoff, K., Stiglitz, J., 2004. After the Big Bang? Obstacles to the emergence of the rule of law in post-Communist societies. American Economic Review 94, 753-763.

Iannotta, G., Nocera, G., Sironi, A., 2007. Ownership structure, risk and performance in the European banking industry. Journal of Banking and Finance 31, 2127 2149.

Jia, C., 2009. The effect of ownership on the prudential behavior of banks: The case of China. Journal of Banking and Finance 33, 77-87.

Knyazeva, A., Knyazeva, D., Stiglitz, J., 2006. Ownership change, institutional development, and performance. Working Paper, New York University.
Lang, L., Ofek, E., Stulz, R., 1996. Leverage, investment, and firm growth. Journal of Financial Economics 40, 3-29.

La Porta, R., Lopez-de-Silanes, F., Shleifer, A., Vishny, R., 1998. Law and finance. Journal of Political Economy 106, 1113-1155

La Porta, R., Lopez-de-Silanes, F., Shelifer, A., Vishny, R., 1999. The quality of government. Journal of Law, Economics, and Organization 15, 222-279.

Li, W., Xu, L., 2004. The impact of privatization and competition in the telecommunications sector around the world. Journal of Law and Economics 47, 395-430.

Lin, X., Zhang, Y., 2009. Bank ownership reform and bank performance in China. Journal of Banking and Finance 33, 20-29.

Megginson, W., Nash, R., Van Randenborgh, M., 1994. The financial and operating performance of newly privatized firms: An international empirical analysis. Journal of Finance 49, 403-452.

Moody's Investors Service, 2003. Sovereign bond defaults, rating transitions, and recoveries (1985-2002). Working Paper, Moody's Investors Service.

Noll, R., 2000. Telecommunications Reform in Developing Countries. In: Krueger, A (Ed.), Economic Policy Reform: The Second Stage. University of Chicago Press Chicago.

Rajan, R., Zingales, L., 1998. Financial dependence and growth. American Economic Review 88, 559-586.

Wallsten, S., 2001. An empirical analysis of competition, privatization, and regulation in Africa and Latin America. Journal of Industrial Economics 49, $1-19$. 